TRANSACTIONS OF THE

AMERICAN MATHEMATICAL SOCIETY

Volume 352, Number 7, Pages 3411-3428

S 0002-9947(00)02530-7

Article electronically published on March 21, 2000

\title{
A GENERALIZED BRAUER CONSTRUCTION AND LINEAR SOURCE MODULES
}

\author{
ROBERT BOLTJE AND BURKHARD KÜLSHAMMER
}

\begin{abstract}
For a complete discrete valuation ring $\mathcal{O}$ with residue field $F$, a subgroup $H$ of a finite group $G$ and a homomorphism $\varphi: H \rightarrow \mathcal{O}^{\times}$, we define a functor $V \mapsto \bar{V}(H, \varphi)$ from the category of $\mathcal{O} G$-modules to the category of $F N_{G}(H, \varphi)$-modules and investigate its behaviour with respect to linear source modules.
\end{abstract}

\section{INTRODUCTION}

Let $\mathcal{O}$ be a complete discrete valuation ring with residue field $F=\mathcal{O} / \pi \mathcal{O}$ of characteristic $p>0$, and let $G$ be a finite group. For a $p$-subgroup $P$ of $G, \mathrm{R}$. Brauer defined a homomorphism of algebras $\mathrm{Br}_{P}: Z(F G) \rightarrow Z\left(F C_{G}(P)\right)$ between the centers of the corresponding group algebras (see CR87, §58]). This Brauer homomorphism plays an important role in representation theory, in particular for the formulation and proof of Brauer's three main theorems on blocks.

The Brauer homomorphism was later generalized from group algebras to modules (see $\left[\mathrm{Br}\right.$ ). If $V$ is an $\mathcal{O} G$-lattice and $H$ is a subgroup of $G$, then $V^{H}$ denotes the set of fixed points of $H$ on $V, \operatorname{tr}_{I}^{H}: V^{I} \rightarrow V^{H}$ denotes the trace map, for $I \leqslant H$, and

$$
V(H)=V^{H} /\left(\sum_{I<H} \operatorname{tr}_{I}^{H}\left(V^{I}\right)+\pi V^{H}\right)
$$

is called the Brauer construction. It is known that $V(H)=0$ if $H$ is not a $p$-group. When $H=P$ is a $p$-group and $V$ is the group algebra $\mathcal{O} G$ endowed with the conjugation action then $V(P)$ can be identified with the group algebra $F C_{G}(P)$, and the canonical map $V^{P} \rightarrow V(P)$ restricts to the classical Brauer homomorphism $\operatorname{Br}_{P}: Z(F G) \rightarrow Z\left(F C_{G}(P)\right)$, composed with the reduction map $Z(\mathcal{O} G) \rightarrow Z(F G)$.

In this paper we extend the applicability of the Brauer construction further, in two directions. First we modify the definition of $V(H)$ in order to get nontrivial results also in cases where $H$ is not a $p$-group. This is achieved by setting

$$
\overline{\bar{V}}(H):=V^{H} /\left(\sum_{\substack{I<H \\ p \mid[H: I]}} \operatorname{tr}_{I}^{H}\left(V^{I}\right)+\pi V^{H}\right)
$$

Then $\overline{\bar{V}}(H)=V(H)$ whenever $H$ is a $p$-group, but $\overline{\bar{V}}(H) \neq 0$ also in many cases where $H$ is not a $p$-group.

Received by the editors April 28, 1998.

2000 Mathematics Subject Classification. Primary 20C11, 20C20.

The first author's research was supported by the DFG. 
Our second modification takes into account homomorphisms $\varphi: H \rightarrow \mathcal{O}^{\times}$. For such a homomorphism $\varphi$, we can consider a generalized fixed point space

$$
V^{(H, \varphi)}:=\{v \in V \mid h v=\varphi(h) v \text { for } h \in H\},
$$

a generalized trace map

$$
\operatorname{tr}_{(I, \psi)}^{(H, \varphi)}: V^{(I, \psi)} \rightarrow V^{(H, \varphi)}, \quad v \mapsto \sum_{h \in H / I} \varphi\left(h^{-1}\right) h v,
$$

whenever $(I, \psi) \leqslant(H, \varphi)$, i.e. $I \leqslant H$ and $\psi=\left.\varphi\right|_{I}$, and a generalized Brauer construction

$$
\overline{\bar{V}}(H, \varphi):=V^{(H, \varphi)} /\left(\sum_{\substack{(I, \psi)<(H, \varphi) \\ p[[H: I]}} \operatorname{tr}_{(I, \psi)}^{(H, \varphi)}\left(V^{(I, \psi)}\right)+\pi V^{(H, \varphi)}\right) .
$$

Just as the "classical" Brauer construction $V(P)$ is most useful with trivial source modules, our modified Brauer construction $\overline{\bar{V}}(H, \varphi)$ is most useful with linear source modules. We show that, for a linear source module $V$, the dimension of $\overline{\bar{V}}(H, \varphi)$ counts the multiplicity of the rank one module $\mathcal{O}_{\varphi}$ as a direct summand of $\operatorname{Res}_{H}^{G}(V)$ and that the dimensions of $\overline{\bar{V}}(H, \varphi)$ determine $V$. We show also that, when $V$ has vertex $P$ and source $\mathcal{O}_{\psi}$ for a homomorphism $\psi: P \rightarrow \mathcal{O}^{\times}$, then $\overline{\bar{V}}(P, \psi)$ coincides with the reduction $\bmod \pi$ of the Green correspondent $W$ of $V$ in $N_{G}(P, \psi)$. As a consequence, we obtain that the functor $V \mapsto \overline{\bar{V}}(P, \psi)$ induces a one-to-one correspondence between the set of isomorphism classes of indecomposable linear source $\mathcal{O} G$-modules with vertex $P$ and source $\mathcal{O}_{\psi}$ and the set of isomorphism classes of indecomposable projective $F\left[N_{G}(P, \psi) / P\right]$-modules.

A nontrivial $p$-group $P$ will, in general, have many homomorphisms $P \rightarrow \mathcal{O}^{\times}$ but only one homomorphism $P \rightarrow F^{\times}$. In order to avoid such phenomena, we study the reduction of linear source modules modulo high powers $\pi^{n}$. We show that, for sufficiently large $n$, reduction modulo $\pi^{n}$ induces an injection (but not a surjection, in general) from the set of isomorphism classes of indecomposable linear source modules over $\mathcal{O}$ into the set of isomorphism classes of indecomposable linear source modules over $\mathcal{O} / \pi^{n} \mathcal{O}$. This injection preserves vertices, sources and Green correspondents. We also show that it suffices to choose $n$ in such a way that $\pi^{n} \mathcal{O}=p \mathcal{O}$ if $p$ is odd, and $\pi^{n} \mathcal{O}=4 \mathcal{O}$ if $p=2$. In this way we obtain an explicit form of Maranda's theorem (cf. [CR81, §30]).

Part of this article was written while the first author was visiting the University of Chicago to which he is grateful for the hospitality he enjoyed.

\section{Notations AND PRELIMINARIES}

1.1. Throughout this article, $G$ denotes a finite group and $E$ its trivial subgroup. Moreover, $R$ always denotes a commutative ring, $R^{\times}$its group of units, $J(R)$ its Jacobson radical, and $R G$ the group algebra of $G$ over $R$. We set $\hat{G}(R):=$ $\operatorname{Hom}\left(G, R^{\times}\right)$, which is an abelian group under multiplication, and we denote by $\mathcal{M}_{G}(R)$ the set of all pairs $(H, \varphi)$, where $H \leqslant G$ and $\varphi \in \hat{H}(R)$. Note that $\mathcal{M}_{G}(R)$ is a partially ordered set, with $(I, \psi) \leqslant(H, \varphi)$ if and only if $I \leqslant H$ and $\psi=\left.\varphi\right|_{I}$. Moreover, $\mathcal{M}_{G}(R)$ is a $G$-set by conjugation: For $g \in G$ and $(H, \varphi) \in \mathcal{M}_{G}(R)$ let ${ }^{g}(H, \varphi):=\left({ }^{g} H,{ }^{g} \varphi\right)$, with ${ }^{g} H:=g H g^{-1}$ and $\left({ }^{g} \varphi\right)(x):=\varphi\left(g^{-1} x g\right)$ for $x \in{ }^{g} H$. By $N_{G}(H, \varphi)$ we denote the stabilizer of $(H, \varphi)$ in $G$. Note that the $G$-action on $\mathcal{M}_{G}(R)$ 
respects the partial order. For a prime $p$ we denote by $\mathcal{M}_{G}(R)_{p} \subseteq \mathcal{M}_{G}(R)$ the subset consisting of those pairs whose first component is a $p$-group. For $\varphi \in \hat{G}(R)$ we denote by $R_{\varphi}$ the $R G$-module which has $R$ itself as underlying $R$-module and carries the $G$-action given by $g \cdot \alpha=\varphi(g) \alpha$ for $g \in G$ and $\alpha \in R$. If $\varphi=1$ is the trivial map, we just write $R$ instead of $R_{\varphi}$. Note that $\varphi \mapsto R_{\varphi}$ induces a bijection between $\hat{G}(R)$ and the set of isomorphism classes of $R G$-modules which are free of rank one as $R$-modules.

If $\Lambda$ is an $R$-order, we denote by ${ }_{\Lambda}$ mod the category of left $\Lambda$-modules which are finitely generated as $R$-modules, and by ${ }_{\Lambda}$ lat $\subseteq{ }_{\Lambda}$ mod the full subcategory of $\Lambda$-lattices. These are the finitely generated $\Lambda$-modules which are projective as $R$ modules. If $V$ is a free $R$-module, we denote its rank by $\operatorname{rk}_{R}(V)$. For two $\Lambda$-modules $V$ and $W$ we write $V \mid W$ if $V$ is isomorphic to a direct summand of $W$.

By $\mathbb{N}, \mathbb{N}_{0}$, and $\mathbb{Z}$ we denote the set of positive, non-negative, and all integers, and by $\mathbb{Q}$ the field of rational numbers. For a prime $p$, we write $\mathbb{Z}_{p}$ and $\mathbb{Q}_{p}$ for the ring of $p$-adic integers and the field of $p$-adic numbers, the completions of $\mathbb{Z}$ and $\mathbb{Q}$ with respect to the $p$-adic valuation.

1.2. Definition. For $V \in{ }_{R G} \bmod$ and any $(H, \varphi) \in \mathcal{M}_{G}(R)$ let

$$
V^{(H, \varphi)}:=\{v \in V \mid h v=\varphi(h) v \text { for all } h \in H\}
$$

denote the $R$-submodule of $(H, \varphi)$-fixed points of $V$. Obviously, for $g \in G$ and $(I, \psi) \leqslant(H, \varphi)$ in $\mathcal{M}_{G}(R)$, one has

$$
V^{g}(H, \varphi)=g V^{(H, \varphi)}, \quad V^{(H, \varphi)} \subseteq V^{(I, \psi)}, \quad V^{(E, 1)}=V .
$$

Thus $V^{(H, \varphi)}$ can be considered as an $R N_{G}(H, \varphi)$-submodule of $V$.

For $(I, \psi) \leqslant(H, \varphi)$ in $\mathcal{M}_{G}(R)$ we define the trace map

$$
\operatorname{tr}_{(I, \psi)}^{(H, \varphi)}: V^{(I, \psi)} \longrightarrow V^{(H, \varphi)}, \quad v \mapsto \sum_{h \in H / I} \varphi\left(h^{-1}\right) h v,
$$

where $h$ runs through a set of representatives for $H / I$. It is easy to check that the sum does not depend on the chosen representatives and that it is an $(H, \varphi)$-fixed point. The following properties of the trace map are immediate.

1.3. Lemma. Let $V \in R G \bmod$, and let $(J, \mu) \leqslant(I, \psi) \leqslant(H, \varphi)$ and $(L, \lambda) \leqslant$ $(H, \varphi)$ be in $\mathcal{M}_{G}(R)$.

(a) For $g \in G$ and $v \in V^{(I, \psi)}$ one has

$$
g \operatorname{tr}_{(I, \psi)}^{(H, \varphi)}(v)=\operatorname{tr}_{g_{(I, \psi)}}^{g(H, \varphi)}(g v) .
$$

Thus the image $V_{(I, \psi)}^{(H, \varphi)}$ of $V^{(I, \psi)}$ under $\operatorname{tr}_{(I, \psi)}^{(H, \varphi)}$ is an $R\left[N_{G}(H, \varphi) \cap N_{G}(I, \psi)\right]$ submodule of $V^{(H, \varphi)}$.

(b) One has $\operatorname{tr}_{(J, \mu)}^{(H, \varphi)}=\operatorname{tr}_{(I, \psi)}^{(H, \varphi)} \circ \operatorname{tr}_{(J, \mu)}^{(I, \psi)}$.

(c) If $V=V_{1} \oplus V_{2}$ is a decomposition into $R H$-submodules, then

$$
V^{(H, \varphi)}=V_{1}^{(H, \varphi)} \oplus V_{2}^{(H, \varphi)} \quad \text { and } \quad V_{(I, \psi)}^{(H, \varphi)}=\left(V_{1}\right)_{(I, \psi)}^{(H, \varphi)} \oplus\left(V_{2}\right)_{(I, \psi)}^{(H, \varphi)}
$$

as $R$-modules.

(d) For each $v \in V^{(H, \varphi)}$ one has

$$
\operatorname{tr}_{(I, \psi)}^{(H, \varphi)}(v)=[H: I] \cdot v .
$$


(e) For $v \in V^{(I, \psi)}, h \in H$ and $x \in L \cap{ }^{h} I$ one has $x h v=\varphi(x) h v$ and

$$
\operatorname{tr}_{(I, \psi)}^{(H, \varphi)}(v)=\sum_{h \in L \backslash H / I} \varphi\left(h^{-1}\right) \operatorname{tr}_{(L \cap h}^{(L, \lambda)}(h, \lambda) \text {. }
$$

1.4. We call an $R G$-module $V$ monomial if there exists a monomial decomposition $V=\bigoplus_{x \in X} V_{x}$ of $V$ into $R$-submodules $V_{x}$ which are free of rank one over $R$ and permuted under the action of $G$. Of course, this is equivalent to saying that $V$ is isomorphic to a finite direct sum of modules of the form $\operatorname{Ind}_{H}^{G}\left(R_{\varphi}\right)$ for various $(H, \varphi) \in \mathcal{M}_{G}(R)$. If $V=\bigoplus_{x \in X} V_{x}$ is a monomial decomposition, then the bijection $x \mapsto V_{x}$ induces an action of $G$ on $X$. By $G_{x}$ we denote the stabilizer in $G$ of an element $x \in X$, and by $\varphi_{x}: G_{x} \rightarrow R^{\times}$we denote the homomorphism by which $G_{x}$ acts on $V_{x}$. Note that $G_{g_{x}}={ }^{g} G_{x}$ and $\varphi_{g_{x}}={ }^{g} \varphi_{x}$ for $g \in G$. If $H \leqslant G$, we set $H_{x}:=H \cap G_{x}$ and often write $\varphi_{x}$ instead of $\left.\varphi_{x}\right|_{H_{x}}$. For any subset $A \subseteq R$, let

$$
\operatorname{Ann}_{R}(A):=\{\alpha \in R \mid \alpha \beta=0 \text { for all } \beta \in A\}
$$

denote the annihilator ideal of $A$ in $R$. For $(H, \varphi),(I, \psi) \in \mathcal{M}_{G}(R)$ we will have to consider the ideal

$$
\mathfrak{a}_{(H, \varphi)}^{(I, \psi)}:=\operatorname{Ann}_{R}(\{\varphi(h)-\psi(h) \mid h \in H \cap I\})
$$

of $R$. The following result will be used repeatedly.

1.5. Lemma. Let $V \in R G$ mod be monomial with monomial decomposition $V=$ $\bigoplus_{x \in X} V_{x}$, and let $(H, \varphi) \in \mathcal{M}_{G}(R)$. Then

$$
V^{(H, \varphi)}=\bigoplus_{x \in H \backslash X} \operatorname{tr}_{\left(H_{x},\left.\varphi\right|_{H_{x}}\right)}^{(H, \varphi)}\left(\mathfrak{a}_{\left(H_{x}, \varphi_{x}\right)}^{(H, \varphi)} \cdot V_{x}\right),
$$

where $x$ runs through a set of representatives for the $H$-orbits of $X$. In particular, if $R$ is an integral domain, one has

$$
V^{(H, \varphi)}=\bigoplus_{\substack{\left.x \in H \backslash X \\ \varphi\right|_{x}=\varphi_{x}}} \operatorname{tr}_{\left(H_{x}, \varphi_{x}\right)}^{(H, \varphi)}\left(V_{x}\right) .
$$

Proof. For $x \in X, v \in V_{x}, \alpha \in \mathfrak{a}_{\left(H_{x}, \varphi_{x}\right)}^{\left(H,{ }_{x}\right)}$, and $h \in H_{x}$ we have

$$
h(\alpha v)=\alpha(h v)=\alpha \varphi_{x}(h) v=\alpha \varphi(h) v=\varphi(h)(\alpha v) .
$$

Therefore, $\alpha v$ is an $\left(H_{x},\left.\varphi\right|_{H_{x}}\right)$-fixed point. Moreover, it is easy to check that the $R$-module

$$
\operatorname{tr}_{\left(H_{x},\left.\varphi\right|_{H_{x}}\right)}^{\left.(H,)_{(}\right)}\left(\mathfrak{a}_{\left(H_{x}, \varphi_{x}\right)}^{(H, \varphi)} \cdot V_{x}\right)
$$

does not depend on the choice of $x$ within its $H$-orbit. The sum on the right hand side in the first equation of the lemma is a direct sum, since the single $R$-modules have support in pairwise distinct $H$-orbits of $X$. So, it suffices to show that $V^{(H, \varphi)}$ is contained in the right hand side. Let $v \in V^{(H, \varphi)}$, and write $v=\sum_{x \in X} v_{x}$ with $v_{x} \in V_{x}$ for $x \in X$. Then, for $h \in H$, we have

$$
\sum_{x \in X} \varphi(h) v_{x}=\varphi(h) v=h v=\sum_{x \in X} h v_{x} .
$$


Comparing components, we conclude that $\varphi(h) v_{h x}=h v_{x}$ for $x \in X$. In particular, $\varphi(h) v_{x}=\varphi_{x}(h) v_{x}$ for $h \in H_{x}$, so $v_{x} \in \mathfrak{a}_{\left(H_{x}, \varphi_{x}\right)}^{(H, \varphi)} \cdot V_{x}$. Thus

$$
v=\sum_{x \in H \backslash X} \sum_{h \in H / H_{x}} v_{h x}=\sum_{x \in H \backslash X} \sum_{h \in H / H_{x}} \varphi\left(h^{-1}\right) h v_{x}=\sum_{x \in H \backslash X} \operatorname{tr}_{\left(H_{x},\left.\varphi\right|_{H_{x}}\right)}^{(H, \varphi)}\left(v_{x}\right),
$$

and the lemma is proved.

The following corollary is now immediate.

1.6. Corollary. Let $(H, \varphi),(I, \psi) \in \mathcal{M}_{G}(R)$. Then

$$
\left(\operatorname{Ind}_{I}^{G}\left(R_{\psi}\right)\right)^{(H, \varphi)}=\bigoplus_{g \in H \backslash G / I} \operatorname{tr}_{\left(H \cap{ }^{I} I, \varphi\right)}^{(H, \varphi)}\left(g \otimes_{R I} \mathfrak{a}_{(H, \varphi)}^{g(I, \psi)}\right) .
$$

In particular, if $R$ is an integral domain, one has

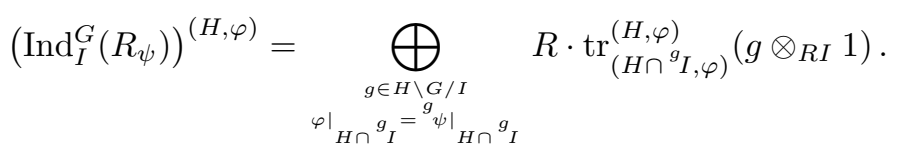

1.7. Remark. We note that $\operatorname{Hom}_{R H}\left(R_{\varphi}, V\right) \cong V^{(H, \varphi)}$ for each $V \in R H$ mod under the map $f \mapsto f(1)$. Together with the adjointness of induction and restriction this yields an isomorphism of $R$-modules

$$
\begin{aligned}
\operatorname{Hom}_{R G}\left(\operatorname{Ind}_{H}^{G}\left(R_{\varphi}\right), \operatorname{Ind}_{I}^{G}\left(R_{\psi}\right)\right) & \rightarrow \bigoplus_{g \in H \backslash G / I} \operatorname{tr}_{\left(H \cap{ }^{g} I, \varphi\right)}^{(H, \varphi)}\left(g \otimes_{R I} \mathfrak{a}_{(H, \varphi)}^{g}(I, \psi)\right. \\
f & \mapsto f\left(1 \otimes_{R H} 1\right) .
\end{aligned}
$$

Thus Corollary 1.6 can also be used to compute Hom-sets and endomorphism rings of monomial modules.

\section{The Generalized Brauer Construction}

2.1. Throughout this section we fix a complete discrete valuation ring $\mathcal{O}$ with field of fractions $K$ of characteristic 0 and with residue field $F$ of prime characteristic $p$. Let $v_{K}: K^{\times} \rightarrow \mathbb{Z}$ denote the surjective valuation with valuation ring $\mathcal{O}$ and let $\pi \in \mathcal{O}$ be an element with $v_{K}(\pi)=1$. Then $\mathcal{O}$ is a local principal ideal domain and each ideal of $\mathcal{O}$ is of the form $\pi^{n} \mathcal{O}$ with $n \in \mathbb{N}_{0} \cup\{\infty\}$, where $\pi^{\infty}:=0$. We may assume that $\mathbb{Z}_{p} \subseteq \mathcal{O}$ and $\mathbb{Q}_{p} \subseteq K$ as the closures of $\mathbb{Z}$ and $\mathbb{Q}$. We denote by $e=e_{K / \mathbb{Q}_{p}}$ the ramification index of $K / \mathbb{Q}_{p}$. Thus $v_{K}(p)=e$ or, equivalently, $p \mathcal{O}=\pi^{e} \mathcal{O}$. We set $k:=\mathcal{O} / p \mathcal{O}$ and denote by ${ }^{-}\left(\right.$resp. $\left.{ }^{\circ}\right)$ the reduction modulo $p \mathcal{O}$ (resp. $\pi \mathcal{O}$ or $\bar{\pi} k$ ).

2.2. Definition. For $V \in \mathcal{O}_{G} \bmod$ and $(H, \varphi) \in \mathcal{M}_{G}(\mathcal{O})$ we set

$$
\bar{V}(H, \varphi):=V^{(H, \varphi)} /\left(\sum_{\substack{(I, \psi)<(H, \varphi) \\ p \mid[H: I]}} V_{(I, \psi)}^{(H, \varphi)}+p V^{(H, \varphi)}\right)
$$

and

$$
\overline{\bar{V}}(H, \varphi):=V^{(H, \varphi)} /\left(\sum_{\substack{(I, \psi)<(H, \varphi) \\ p\lceil[H: I]}} V_{(I, \psi)}^{(H, \varphi)}+\pi V^{(H, \varphi)}\right) .
$$


We call $\bar{V}(H, \varphi) \in{ }_{k N_{G}(H, \varphi)} \bmod$ and $\overline{\bar{V}}(H, \varphi) \in{ }_{F N_{G}(H, \varphi)} \bmod$ the generalized Brauer construction of $V$ with respect to $(H, \varphi)$. Moreover, we call the canonical maps

$$
\overline{\mathrm{Br}}_{(H, \varphi)}: V^{(H, \varphi)} \rightarrow \bar{V}(H, \varphi)
$$

and

$$
\overline{\overline{\mathrm{Br}}}_{(H, \varphi)}: V^{(H, \varphi)} \rightarrow \overline{\bar{V}}(H, \varphi)
$$

the generalized Brauer maps with respect to $(H, \varphi)$.

2.3. Remark. (a) It is clear from Definition 2.2 that the generalized Brauer construction induces functors

$$
\left\ulcorner(H, \varphi):{ }_{\mathcal{O} G} \bmod \rightarrow{ }_{k N_{G}(H, \varphi)} \bmod \right.
$$

and

$$
\bar{\digamma}(H, \varphi): \mathcal{O} G_{G} \bmod \rightarrow{ }_{F N}(H, \varphi) \bmod
$$

which commute with direct sums. Note that, by definition, the $F N_{G}(H, \varphi)$-module $\overline{\bar{V}}(H, \varphi)$ arises from $\bar{V}(H, \varphi)$ by reduction modulo $\bar{\pi} k$.

(b) Let $\varphi=1$ be the trivial homomorphism. If $H$ is a $p$-subgroup of $G$, then $\overline{\bar{V}}(H, \varphi)$ in Definition 2.2 coincides with the usual Brauer construction, cf. $\mathrm{Br}$ ] or Th, §11]. If $H$ is not a $p$-subgroup of $G$, then the usual Brauer construction with respect to $H$ is identically zero whereas $\overline{\bar{V}}(H, \varphi)$ is not, in general.

(c) Note that by Lemma $1.3(\mathrm{~d})$, one has

$$
p V^{(H, \varphi)} \subseteq \sum_{\substack{(I, \psi)<(H, \varphi) \\ p \mid[H: I]}} V_{(I, \psi)}^{(H, \varphi)},
$$

if $p$ divides $|H|$. Note also that $\bar{V}(H, \varphi)=V^{(H, \varphi)} / p V^{(H, \varphi)}$ in the case that $H$ is a $p^{\prime}$-group. We will see that $\bar{V}(H, \varphi)$ is free as a $k$-module if $V$ is monomial. There is no immediate need to go over to $\overline{\bar{V}}(H, \varphi)$ instead of using $\bar{V}(H, \varphi)$. In fact, the inclusion in (2.3.a) indicates that the construction $\bar{V}(H, \varphi)$ is more natural than $\overline{\bar{V}}(H, \varphi)$.

2.4. Proposition. Let $V$ be a monomial $\mathcal{O} G$-module with monomial decomposition $V=\bigoplus_{x \in X} V_{x}$, and let $(H, \varphi) \in \mathcal{M}_{G}(\mathcal{O})$. Then

$$
\sum_{\substack{(I, \psi)<(H, \varphi) \\ p \mid[H: I]}} V_{(I, \psi)}^{(H, \varphi)}+p V^{(H, \varphi)}=\bigoplus_{\substack{x \in H \backslash X \\ \varphi H_{x}=\varphi_{x} \\ p \mid[H: H x]}} \operatorname{tr}_{\left(H_{x}, \varphi_{x}\right)}^{\left(H, \varphi_{x}\right)}\left(V_{x}\right) \oplus \bigoplus_{\substack{x \in H \backslash X \\ \varphi H_{x}=\varphi_{x} \\ p \nmid\left[H: H_{x}\right]}} p \cdot \operatorname{tr}_{\left(H_{x}, \varphi_{x}\right)}^{\left(H, \varphi_{x}\right)}\left(V_{x}\right) .
$$

Thus $\bar{V}(H, \varphi)$ is a monomial $k N_{G}(H, \varphi)$-module with monomial decomposition

$$
\bar{V}(H, \varphi)=\bigoplus_{\substack{\left.x \in H \backslash X \\ \varphi\right|_{x}=\varphi_{x} \\ p \chi\left[H: H_{x}\right]}} \bar{V}(H, \varphi)_{\bar{x}}
$$

where $\bar{x}$ denotes the $H$-orbit of $x$ and $\bar{V}(H, \varphi)_{\bar{x}}:=\overline{\operatorname{Br}}_{(H, \varphi)}\left(\operatorname{tr}_{\left(H_{x}, \varphi_{x}\right)}^{(H, \varphi)}\left(V_{x}\right)\right)$, for $x \in X$. The subgroup $N_{G}(H, \varphi)$ of $G$ permutes the $k$-submodules $\bar{V}(H, \varphi)_{\bar{x}}$ of $\bar{V}(H, \varphi)$ in the same way as the $H$-orbits $\bar{x}$. Thus $N_{G}(H, \varphi)_{\bar{x}}=N_{G}(H, \varphi)_{x} H$, 
and $N_{G}(H, \varphi)_{\bar{x}}$ acts on $\bar{V}(H, \varphi)_{\bar{x}}$ via the homomorphism $\overline{\varphi_{x} \varphi}: N_{G}(H, \varphi)_{\bar{x}} \rightarrow k^{\times}$ defined by $\left(\varphi_{x} \varphi\right)(a b):=\varphi_{x}(a) \varphi(b)$ for $a \in N_{G}(H, \varphi)_{x}, b \in H$.

Proof. We recall that

$$
V^{(H, \varphi)}=\bigoplus_{\substack{\left.x \in H \backslash X \\ \varphi\right|_{H x}=\varphi_{x}}} \operatorname{tr}_{\left(H_{x}, \varphi_{x}\right)}^{(H, \varphi)}\left(V_{x}\right),
$$

where each summand is free of rank one over $\mathcal{O}$. Therefore the right hand side of the first equation to be proved is indeed a direct sum contained in the left hand side, and $p V^{(H, \varphi)}$ is contained in the right hand side. Let $(I, \psi)<(H, \varphi)$ be such that $p \mid[H: I]$, and let $v \in V_{(I, \psi)}^{(H, \varphi)}$. Then $v$ is a sum of elements of the form

$$
w_{x}=\operatorname{tr}_{(I, \psi)}^{(H, \varphi)}\left(\operatorname{tr}_{\left(I_{x}, \varphi_{x}\right)}^{(I, \psi)}\left(v_{x}\right)\right)=\operatorname{tr}_{\left(I_{x}, \varphi_{x}\right)}^{(H, \varphi)}\left(v_{x}\right)=\left[H_{x}: I_{x}\right] \operatorname{tr}_{\left(H_{x}, \varphi_{x}\right)}^{(H, \varphi)}\left(v_{x}\right),
$$

where $v_{x} \in V_{x}$ and $\left.\psi\right|_{I_{x}}=\varphi_{x}$. If $p \mid\left[H_{x}: I_{x}\right]$ then $w_{x} \in p V^{(H, \varphi)}$, which is contained in the right hand side. Thus suppose that $p \nmid\left[H_{x}: I_{x}\right]$. Then $p \mid\left[H: H_{x}\right]$, for otherwise we would have $p \nmid\left[H: I_{x}\right]$, contradicting our assumption $p \mid[H: I]$. Therefore $w_{x}$ is also contained in the right hand side in this case.

From this we get the decomposition of $\bar{V}(H, \varphi)$ into its $k$-submodules $\bar{V}(H, \varphi)_{\bar{x}}$ which are free of rank one over $k$. It is immediate that these submodules are permuted according to the canonical action of $N_{G}(H, \varphi)$ on $H \backslash X$. A Frattini argument shows that $N_{G}(H, \varphi)_{\bar{x}}=N_{G}(H, \varphi)_{x} H$. If $\left.\varphi\right|_{H_{x}}=\varphi_{x}$, then, for $v \in V_{x}$, $a \in N_{G}(H, \varphi)_{x}$ and $b \in H$, we have

$$
\begin{aligned}
\operatorname{abtr}_{\left(H_{x}, \varphi_{x}\right)}^{(H, \varphi)}(v) & =a \varphi(b) \operatorname{tr}_{\left(H_{x}, \varphi_{x}\right)}^{(H, \varphi)}(v)=\varphi(b) \operatorname{tr}_{\left({ }^{a} H_{x},{ }^{a} \varphi_{x}\right)}^{(H, \varphi)}(a v) \\
& =\varphi_{x}(a) \varphi(b) \operatorname{tr}_{\left(H_{a x},{ }^{a} \varphi \mid H_{a x}\right)}^{(H, \varphi)}(v)=\varphi_{x}(a) \varphi(b) \operatorname{tr}_{\left(H_{x},\left.\varphi\right|_{H_{x}}\right)}^{(H, \varphi)}(v) \\
& =\varphi_{x}(a) \varphi(b) \operatorname{tr}_{\left(H_{x}, \varphi_{x}\right)}^{(H, \varphi)}(v) .
\end{aligned}
$$

The result follows.

For any $V \in \mathcal{O}_{G}$ lat and any $(H, \varphi) \in \mathcal{M}_{G}(\mathcal{O})$ let $m_{(H, \varphi)}(V)$ denote the multiplicity with which the $\mathcal{O} H$-module $\mathcal{O}_{\varphi}$ occurs as a direct summand in $\operatorname{Res}_{H}^{G}(V)$. Our next aim is to show that $m_{(H, \varphi)}(V)$ is related to the $k$-rank of $\bar{V}(H, \varphi)$ whenever $V$ is monomial.

The following terminology will also be useful. For $V \in \mathcal{O}_{G}$ lat, we will call an

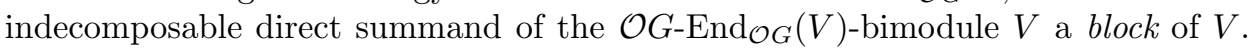
The motivation for this terminology comes from the fact that, when $V$ is the group algebra $\mathcal{O} G$, considered as a left $\mathcal{O} G$-module under multiplication, then $\operatorname{End}_{\mathcal{O} G}(V)$ can be identified with $\mathcal{O} G$, acting by right multiplication, so the blocks of $V$ are just the blocks of $\mathcal{O} G$ in the usual sense.

It is clear that every $V \in \mathcal{O}_{G}$ lat has a decomposition

$$
V=V_{1} \oplus \cdots \oplus V_{r}
$$

into blocks $V_{1}, \ldots, V_{r}$. This decomposition gives rise to a decomposition

$$
1_{E}=\mathrm{id}_{V}=e_{1}+\cdots+e_{r}
$$

of the identity element $1_{E}=\operatorname{id}_{V}$ of $E:=\operatorname{End}_{\mathcal{O} G}(V)$ into pairwise orthogonal idempotents $e_{1}, \ldots, e_{r}$ in $E$, where each $e_{i}$ is the projection onto the direct summand $V_{i}$. One checks that $e_{1}, \ldots, e_{r}$ are contained in the center of $E$. It follows that 
$e_{1}, \ldots, e_{r}$ are just the block idempotents of $E$. Thus we obtain a corresponding decomposition

$$
E=\operatorname{End}_{\mathcal{O} G}(V)=E_{1} \oplus \cdots \oplus E_{r}
$$

of $E$ into blocks $E_{1}=e_{1} E, \ldots, E_{r}=e_{r} E$. In this way the blocks of the $\mathcal{O} G$-lattice $V$ correspond to the blocks of the $\mathcal{O}$-order $E$. Since $V_{i}=E_{i} \cdot V$ for $i=1, \ldots, r$, the blocks $V_{1}, \ldots, V_{r}$ are uniquely determined by $V$ up to their order (and not just up to isomorphism). It is easy to see that if $W$ is a direct summand of $V$ as $\mathcal{O} G$-module, then also $W=e_{1} W \oplus \cdots \oplus e_{r} W$ is a decomposition of (not necessarily indecomposable) $\mathcal{O} G$-End $\operatorname{En}_{\mathcal{G}}(W)$-bimodules.

2.5. Lemma. Let $\varphi \in \hat{G}(\mathcal{O})$ and $(I, \psi) \in \mathcal{M}_{G}(\mathcal{O})$. Then

$$
m_{(G, \varphi)}\left(\operatorname{Ind}_{I}^{G}\left(\mathcal{O}_{\psi}\right)\right)= \begin{cases}1, & \text { if } \psi=\left.\varphi\right|_{I} \text { and } p \nmid[G: I], \\ 0, & \text { otherwise. }\end{cases}
$$

Moreover, if $m_{(G, \varphi)}\left(\operatorname{Ind}_{I}^{G}\left(\mathcal{O}_{\psi}\right)\right)=1$, then $\operatorname{Ind}_{I}^{G}\left(\mathcal{O}_{\psi}\right)^{(G, \varphi)}$ is a block of $\operatorname{Ind}_{I}^{G}\left(\mathcal{O}_{\psi}\right)$.

Proof. Since

$$
\operatorname{Hom}_{\mathcal{O} G}\left(\operatorname{Ind}_{I}^{G}\left(\mathcal{O}_{\psi}\right), \mathcal{O}_{\varphi}\right) \cong \operatorname{Hom}_{\mathcal{O} I}\left(\mathcal{O}_{\psi}, \mathcal{O}_{\left.\varphi\right|_{I}}\right)
$$

the multiplicity $m_{(G, \varphi)}\left(\operatorname{Ind}_{I}^{G}\left(\mathcal{O}_{\psi}\right)\right)$ can only assume the values 0 and 1 , and it is zero if $\psi \neq\left.\varphi\right|_{I}$. So we assume $\psi=\left.\varphi\right|_{I}$ from now on. If $\mathcal{O}_{\varphi} \mid \operatorname{Ind}_{I}^{G}\left(\mathcal{O}_{\psi}\right)$, then $I$ contains a Sylow $p$-subgroup of $G$, since $\mathcal{O}_{\varphi}$ has the Sylow $p$-subgroups of $G$ as vertices. Therefore, $p \nmid[G: I]$. Conversely, assume that $p \nmid[G: I]$. Then the maps

$$
\vartheta: \operatorname{Ind}_{I}^{G}\left(\mathcal{O}_{\psi}\right) \rightarrow \mathcal{O}_{\varphi}, \quad \sum_{g \in G / I} g \otimes_{\mathcal{O} I} \alpha_{g} \mapsto \sum_{g \in G / I} \varphi(g) \alpha_{g}
$$

and

$$
\eta: \mathcal{O}_{\varphi} \rightarrow \operatorname{Ind}_{I}^{G}\left(\mathcal{O}_{\psi}\right), \quad \alpha \mapsto[G: I]^{-1} \sum_{g \in G / I} \varphi\left(g^{-1}\right) g \otimes_{\mathcal{O} I} \alpha
$$

are independent of the choice of the transversal for $G / I$ and $\mathcal{O} G$-linear such that $\vartheta \circ \eta=\operatorname{id}_{\mathcal{O}_{\varphi}}$. Thus $\epsilon:=\eta \circ \vartheta$ is an idempotent in $E:=\operatorname{End}_{\mathcal{O} G}\left(\operatorname{Ind}_{I}^{G}\left(\mathcal{O}_{\psi}\right)\right)$ with image

$$
\epsilon\left(\operatorname{Ind}_{I}^{G}\left(\mathcal{O}_{\psi}\right)\right)=\eta\left(\mathcal{O}_{\varphi}\right) \cong \mathcal{O}_{\varphi}
$$

Hence, we get $\epsilon\left(\operatorname{Ind}_{I}^{G}\left(\mathcal{O}_{\psi}\right)\right)=\operatorname{Ind}_{I}^{G}\left(\mathcal{O}_{\psi}\right)^{(G, \varphi)}$, which is an $\mathcal{O} G$-E-subbimodule of $\operatorname{Ind}_{I}^{G}\left(\mathcal{O}_{\psi}\right)$. It is easy to check that $\operatorname{ker}(\vartheta)=\sum_{g \in G}\left[(g-\varphi(g) 1) \operatorname{Ind}_{I}^{G}\left(\mathcal{O}_{\psi}\right)\right]$. Thus,

$$
\operatorname{Ind}_{I}^{G}\left(\mathcal{O}_{\psi}\right)=\eta\left(\mathcal{O}_{\varphi}\right) \oplus \operatorname{ker}(\vartheta)
$$

is a decomposition of $\mathcal{O} G$-E-bimodules, and the result follows.

The following result is now immediate from Proposition 2.4 and Lemma 2.5.

2.6. Proposition. Let $V$ be a monomial $\mathcal{O} G$-module and $(H, \varphi) \in \mathcal{M}_{G}(\mathcal{O})$. Then

$$
m_{(H, \varphi)}(V)=\operatorname{rk}_{k} \bar{V}(H, \varphi)=\operatorname{dim}_{F} \overline{\bar{V}}(H, \varphi) .
$$


2.7. Example. If $V \in{ }_{\mathcal{O} G}$ lat is not monomial, then $\bar{V}(H, \varphi)$ need not be free as a $k$-module, and the dimension of $\overline{\bar{V}}(H, \varphi)$ need not be the multiplicity of $\mathcal{O}_{\varphi}$ in $\operatorname{Res}_{H}^{G}(V)$. In fact, let $G=\langle x\rangle$ be the group of order $3, p=3$, let $\mathcal{O}$ contain a primitive third root of unity $\zeta$, let $H=G$, and let $\varphi \in \hat{G}(\mathcal{O})$ be defined by $\varphi(x)=\zeta$. Furthermore, let $V=\left\{\alpha+\beta x+\gamma x^{2} \in \mathcal{O} G \mid \alpha+\beta+\gamma=0\right\}$ be the augmentation ideal of $\mathcal{O} G$. Then $V$ is indecomposable, since its reduction modulo $\pi$ is the radical of $F G$. Moreover, one has $V^{(H, \varphi)}=\mathcal{O} \cdot\left(1+\zeta^{2} x+\zeta x\right)$ and $V_{(E, 1)}^{(H, \varphi)}=(1-\zeta) \mathcal{O} \cdot\left(1+\zeta^{2} x+\zeta x\right)$, so $\bar{V}(H, \varphi) \cong \mathcal{O} /(1-\zeta) \mathcal{O}$ and also $\overline{\bar{V}}(H, \varphi) \cong F$, whereas the multiplicity of $\mathcal{O}_{\varphi}$ in $V$ is zero. Note that, since $(1-\zeta)^{2} \mathcal{O}=3 \mathcal{O}$, the $k$-module $\bar{V}(H, \varphi)$ is not free.

In the next section, we will show that Proposition 2.6] remains valid for $\mathcal{O} G$ lattices which are direct summands of monomial modules.

\section{Linear SOURCE MODUles}

3.1. We continue with the notation introduced in 2.1. In this section we are going to study linear source modules, i.e. direct summands of monomial modules. In view of the preceding section, we will work with the coefficient rings $\mathcal{O}, k$ and $F$. In order to handle these coefficient rings simultaneously, we fix $n \in \mathbb{N} \cup\{\infty\}$ and set $R:=\mathcal{O} / \pi^{n} \mathcal{O}$. Then many of the usual theorems for $\mathcal{O} G$-lattices hold also for $R G$-lattices: The Krull-Schmidt Theorem and the theory of vertices and sources are valid, as well as the Green correspondence and the Higman criterion (see $\mathrm{Be}$, Sections 3.10, 3.12, and Proposition 3.6.4]). Moreover, in special situations also Green's indecomposability theorem holds: Let $I \unlhd H$ be subgroups of $G$ such that $H / I$ is a $p$-group and let $\psi \in \hat{I}(R)$. Then $\operatorname{Ind}_{I}^{H}\left(\bar{R}_{\psi}\right)$ is indecomposable. In fact, if $\operatorname{Ind}_{I}^{H}\left(R_{\psi}\right)$ were decomposable, then so would be its reduction modulo $J(R)$, which is isomorphic to $\operatorname{Ind}_{I}^{H}\left(F_{\bar{\psi}}\right)$. But the latter module certainly is indecomposable by Green's original theorem [Gr. Theorem 8]. An $R G$-lattice is called a linear source $R G$-module (resp. trivial source $R G$-module), if it is a direct sum of indecomposable $R G$-lattices which have sources of $R$-rank one (resp. trivial sources). We write ${ }_{R G}$ triv $\subseteq R G$ lin $\subseteq$ $R G$ lat for the full subcategories of linear source $R G$-modules and trivial source $R G$-modules, respectively. A pair $(P, \psi) \in \mathcal{M}_{G}(R)_{p}$ is called a defect pair of an indecomposable linear source $R G$-module $V$ if $P$ is a vertex and $R_{\psi}$ a source of $V$. Note that the defect pairs of $V$ are unique up to $G$-conjugacy. The following lemma is proved in precisely the same way as in [Bo98, Proposition 1] (resp. [Br, (0.2), (0.4)]).

3.2. Lemma. Let $n \in \mathbb{N} \cup\{\infty\}$ and let $R=\mathcal{O} / \pi^{n} \mathcal{O}$.

(a) For $V \in{ }_{R G}$ lat and a Sylow p-subgroup $P$ of $G$ the following statements are equivalent:

(i) The lattice $V$ is a linear (resp. trivial) source $R G$-module.

(ii) The lattice $\operatorname{Res}_{P}^{G}(V)$ is a monomial (resp. permutation) RP-module.

(iii) The lattice $V$ is isomorphic to a direct summand of a monomial (resp. permutation) $R G$-module.

(b) For $H \leqslant G$, for linear (resp. trivial) source $R G$-modules $V, V^{\prime}$, and for a linear (resp. trivial) source $R H$-module $W$ one has:

(i) The $R G$-lattices $V \oplus V^{\prime}, V \otimes V^{\prime}$, and $V^{*}=\operatorname{Hom}_{R}(V, R)$ are again linear (resp. trivial) source $R G$-modules. 
(ii) The $R H$-lattice $\operatorname{Res}_{H}^{G}(V)$ is a linear (resp. trivial) source $R H$-module.

(iii) The $R G$-lattice $\operatorname{Ind}_{H}^{G}(W)$ is a linear (resp. trivial) source $R G$-module.

(iv) Every direct summand of $V$ is a linear (resp. trivial) source $R G$-module.

Our first main result in this section is a Clifford type theorem for linear source modules. Before we state it, we would like to recall some elementary facts in Clifford theory for which we do not know a suitable reference.

Let $V \in \mathcal{O}_{G}$ lat be indecomposable, and let $H \unlhd G$. We consider the decomposition

$$
\operatorname{Res}_{H}^{G}(V)=V_{1} \oplus \cdots \oplus V_{r}
$$

of $\operatorname{Res}_{H}^{G}(V)$ into blocks $V_{1}, \ldots, V_{r}$. Then, for $g \in G$, we have

$$
V=g V=g V_{1} \oplus \cdots \oplus g V_{r},
$$

where each $g V_{i}$ is also a block of $\operatorname{Res}_{H}^{G}(V)$, as is easily checked. This shows that the action of $G$ on $V$ permutes the blocks of $\operatorname{Res}_{H}^{G}(V)$. Since $V$ is indecomposable, it must permute them transitively. Thus, if we denote the stabilizer of $V_{1}$ in $G$ by $I$, then $V_{1}$ is an $\mathcal{O} I$-lattice such that

$$
\operatorname{Res}_{H}^{G}(V)=\bigoplus_{g \in G / I} g V_{1} \text { and } V \cong \operatorname{Ind}_{I}^{G}\left(V_{1}\right)
$$

3.3. Theorem. Let $V \in \mathcal{O}_{G}$ lin be indecomposable, and let $(H, \varphi) \in \mathcal{M}_{G}(\mathcal{O})$ be such that $H \unlhd G$ and $\mathcal{O}_{\varphi} \mid \operatorname{Res}_{H}^{G}(V)$. Then $V$ has a defect pair $(P, \psi)$ with the following properties:

(i) $P$ is a Sylow p-subgroup of $H P$.

(ii) There exists a homomorphism $\lambda: H P \rightarrow \mathcal{O}^{\times}$such that $\left.\lambda\right|_{H}=\varphi$ and $\left.\lambda\right|_{P}=\psi$; in particular, $\varphi$ is $P$-stable and $H P \leqslant N_{G}(H, \varphi)$.

(iii) $V \cong \operatorname{Ind}_{N_{G}(H, \varphi)}^{G}\left(V^{(H, \varphi)}\right)$ and $V^{(H, \varphi)}$ is an indecomposable linear source $\mathcal{O} N_{G}(H, \varphi)$-module with defect pair $(P, \psi)$.

Proof. Let $(P, \psi)$ be an arbitrary defect pair of $V$. Since $V \mid \operatorname{Ind}_{P}^{G}\left(\mathcal{O}_{\psi}\right)$, there exists an indecomposable direct summand $W$ of $\operatorname{Ind}_{P}^{H P}\left(\mathcal{O}_{\psi}\right)$ such that $V \mid \operatorname{Ind}_{H P}^{G}(W)$. It is immediate that $(P, \psi)$ is a defect pair of $W$ as well. Moreover, we have

$$
\begin{aligned}
& \mathcal{O}_{\varphi}\left|\operatorname{Res}_{H}^{G}(V)\right| \operatorname{Res}_{H}^{G}\left(\operatorname{Ind}_{H P}^{G}(W)\right) \\
& \cong \bigoplus_{g \in H \backslash G / H P} \operatorname{Ind}_{H \cap g(H P)}^{H}\left(\operatorname{Res}_{H \cap{ }^{g}(H P)}^{g}\left({ }^{g} W\right)\right) \cong \bigoplus_{g \in G / H P}{ }^{g} \operatorname{Res}_{H}^{H P}(W) \text {. }
\end{aligned}
$$

After replacing $(P, \psi)$ and $W$ by suitable conjugates we may assume that $\mathcal{O}_{\varphi} \mid$ $\operatorname{Res}_{H}^{H P}(W)$. Then we have

$$
\mathcal{O}_{\varphi}\left|\operatorname{Res}_{H}^{H P}(W)\right| \operatorname{Res}_{H}^{H P}\left(\operatorname{Ind}_{P}^{H P}\left(\mathcal{O}_{\psi}\right)\right) \cong \operatorname{Ind}_{H \cap P}^{H}\left(\mathcal{O}_{\left.\psi\right|_{H \cap P}}\right) .
$$

By Lemma 2.5, this implies that $H \cap P$ is a Sylow $p$-subgroup of $H$, that $\left.\varphi\right|_{H \cap P}=$ $\left.\psi\right|_{H \cap P}$ and that $\operatorname{Ind}_{H \cap P}^{H}\left(\mathcal{O}_{\left.\psi\right|_{H \cap P}}\right)^{(H, \varphi)}$ is a block of $\operatorname{Ind}_{H \cap P}^{H}\left(\mathcal{O}_{\left.\psi\right|_{H \cap P}}\right)$. Therefore $W^{(H, \varphi)}$ is a block of $\operatorname{Res}_{H}^{H P}(W)$, of $\mathcal{O}$-rank 1. Let $I$ denote the stabilizer of $W^{(H, \varphi)}$ in $H P$. By the remarks preceding the theorem we have $W \cong \operatorname{Ind}_{I}^{H P}\left(W^{(H, \varphi)}\right)$; in particular, $W$ is $I$-projective. On the other hand, $W$ has defect pair $(P, \psi)$, where $P$ is a Sylow $p$-subgroup of $H P$ since $H \cap P$ is a Sylow $p$-subgroup of $H$. Hence we have $p \nmid[H P: I] \mid[H P: H]=[P: P \cap H]$, which implies that $I=H P$. Thus $\operatorname{Res}_{H}^{H P}(W)=W^{(H, \varphi)} \cong \mathcal{O}_{\varphi}$; in particular, we have $\operatorname{rk}_{\mathcal{O}} W=1$. So there exists a 
homomorphism $\lambda: H P \rightarrow \mathcal{O}^{\times}$such that $W \cong \mathcal{O}_{\lambda}$. Since $\operatorname{Res}_{H}^{H P}(W) \cong \mathcal{O}_{\varphi}$, we get $\left.\lambda\right|_{H}=\varphi$. Since $\mathcal{O}_{\lambda} \cong W \mid \operatorname{Ind}_{P}^{H P}\left(\mathcal{O}_{\psi}\right)$, Lemma 2.5 implies that $\left.\lambda\right|_{P}=\psi$. Finally, $V \mid \operatorname{Ind}_{H P}^{G}(W) \cong \operatorname{Ind}_{H P}^{G}\left(\mathcal{O}_{\lambda}\right)$ implies that

$$
\operatorname{Res}_{H}^{G}(V) \mid \operatorname{Res}_{H}^{G}\left(\operatorname{Ind}_{H P}^{G}\left(\mathcal{O}_{\lambda}\right)\right) \cong \bigoplus_{g \in G / H P}{ }^{g} \operatorname{Res}_{H}^{H P}\left(\mathcal{O}_{\lambda}\right)=\bigoplus_{g \in G / H P}{ }^{g} \mathcal{O}_{\varphi} .
$$

Thus $\operatorname{Res}_{H}^{G}(V)=\bigoplus_{g \in G / N_{G}(H, \varphi)} g V^{(H, \varphi)}$ and $V \cong \operatorname{Ind}_{N_{G}(H, \varphi)}^{G}\left(V^{(H, \varphi)}\right)$; in particular, the $\mathcal{O} N_{G}(H, \varphi)$-module $V^{(H, \varphi)}$ is indecomposable. Now $V \mid \operatorname{Ind}_{H P}^{G}\left(\mathcal{O}_{\lambda}\right)$, so by Corollary 1.6 we obtain

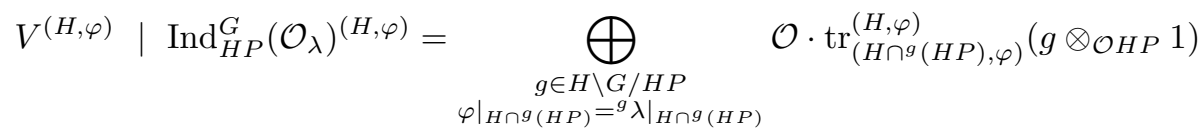

$$
\begin{aligned}
& =\bigoplus_{\substack{g \in G / H P \\
\varphi=\left.{ }^{g} \lambda\right|_{H}}} \mathcal{O}\left(g \otimes_{\mathcal{O} H P} 1\right)=\bigoplus_{g \in N_{G}(H, \varphi) / H P} \mathcal{O}\left(g \otimes_{\mathcal{O} H P} 1\right) \\
& \cong \operatorname{Ind}_{H P}^{N_{G}(H, \varphi)}\left(\mathcal{O}_{\lambda}\right) \mid \operatorname{Ind}_{P}^{N_{G}(H, \varphi)}\left(\mathcal{O}_{\psi}\right) .
\end{aligned}
$$

Thus the $\mathcal{O} N_{G}(H, \varphi)$-module $V^{(H, \varphi)}$ is $P$-projective. Let $(Q, \mu)$ be a defect pair of $V^{(H, \varphi)}$ such that $Q \leqslant P$. Then $V^{(H, \varphi)} \mid \operatorname{Ind}_{Q}^{N_{G}(H, \varphi)}\left(\mathcal{O}_{\mu}\right)$ and

$$
V \cong \operatorname{Ind}_{N_{G}(H, \varphi)}^{G}\left(V^{(H, \varphi)}\right) \mid \operatorname{Ind}_{Q}^{G}\left(\mathcal{O}_{\mu}\right)
$$

so $V$ is $Q$-projective. Since $V$ has defect pair $(P, \psi)$, we conclude that $|P| \leqslant|Q| \leqslant$ $|P|$. Thus $P=Q$, and $(P, \psi)$ is a defect pair of $V^{(H, \varphi)}$.

We get the following consequence.

3.4. Corollary. Let $(H, \varphi) \in \mathcal{M}_{G}(\mathcal{O})$ with $H \unlhd G$, and let $I:=N_{G}(H, \varphi)$. Then the maps $V \mapsto V^{(H, \varphi)}$ and $W \mapsto \operatorname{Ind}_{I}^{G}(W)$ induce mutually inverse bijections between the set of isomorphism classes of indecomposable linear source $\mathcal{O} G$-modules $V$ such that $\mathcal{O}_{\varphi} \mid \operatorname{Res}_{H}^{G}(V)$ and the set of isomorphism classes of indecomposable linear source $\mathcal{O} I$-modules $W$ such that $\mathcal{O}_{\varphi} \mid \operatorname{Res}_{H}^{I}(W)$. Moreover, the map $W \mapsto \operatorname{Ind}_{I}^{G}(W)$ preserves defect pairs.

Proof. Let $V$ be an indecomposable linear source $\mathcal{O} G$-module such that $\mathcal{O}_{\varphi} \mid$ $\operatorname{Res}_{H}^{G}(V)$. Then, by Theorem 3.3, $V^{(H, \varphi)}$ is an indecomposable linear source $\mathcal{O} I$ module, and $V \cong \operatorname{Ind}_{I}^{G}\left(V^{(H, \varphi)}\right)$. Moreover, it is immediate that $\mathcal{O}_{\varphi} \mid \operatorname{Res}_{H}^{I}\left(V^{(H, \varphi)}\right)$.

Conversely, let $W$ be an indecomposable linear source $\mathcal{O} I$-module such that $\mathcal{O}_{\varphi} \mid$ $\operatorname{Res}_{H}^{I}(W)$. Then Theorem 3.3 implies that $W=W^{(H, \varphi)}$. Since $W \mid \operatorname{Res}_{I}^{G}\left(\operatorname{Ind}_{I}^{G}(W)\right)$ there exists an indecomposable direct summand $V$ of $\operatorname{Ind}_{I}^{G}(W)$ such that $W$ | $\operatorname{Res}_{I}^{G}(V)$. Thus $\mathcal{O}_{\varphi}\left|\operatorname{Res}_{H}^{I}(W)\right| \operatorname{Res}_{H}^{G}(V)$. By Theorem 3.3, we have $V \cong$ $\operatorname{Ind}_{I}^{G}\left(V^{(H, \varphi)}\right)$, and

$$
\begin{aligned}
\operatorname{rk}_{\mathcal{O}} V & =[G: I] \mathrm{rk}_{\mathcal{O}} V^{(H, \varphi)} \geqslant[G: I] \mathrm{rk}_{\mathcal{O}} W^{(H, \varphi)} \\
& =[G: I] \mathrm{rk}_{\mathcal{O}} W=\operatorname{rk}_{\mathcal{O}} \operatorname{Ind}_{I}^{G}(W) \geqslant \operatorname{rk}_{\mathcal{O}} V
\end{aligned}
$$

It follows that $\operatorname{Ind}_{I}^{G}(W)=V$ is an indecomposable linear source $\mathcal{O} G$-module and $W^{(H, \varphi)} \cong V^{(H, \varphi)}$; hence $W=W^{(H, \varphi)} \cong V^{(H, \varphi)}=\operatorname{Ind}_{I}^{G}(W)^{(H, \varphi)}$. Finally, the statement about defect pairs is an easy consequence of Theorem 3.3. 
3.5. Theorem. Let $V \in \mathcal{O}_{G}$ lin and $(H, \varphi) \in \mathcal{M}_{G}(\mathcal{O})$. Then $\bar{V}(H, \varphi) \in{ }_{k N_{G}(H, \varphi)}$ lin, $\overline{\bar{V}}(H, \varphi) \in_{F N_{G}(H, \varphi)}$ triv, and

$$
\operatorname{dim}_{F} \overline{\bar{V}}(H, \varphi)=\operatorname{rk}_{k} \bar{V}(H, \varphi)=m_{(H, \varphi)}(V) .
$$

In particular, one has

$$
\overline{\bar{V}}(H, \varphi) \neq 0 \Longleftrightarrow \bar{V}(H, \varphi) \neq 0 \Longleftrightarrow \mathcal{O}_{\varphi} \mid \operatorname{Res}_{H}^{G}(V) .
$$

Proof. By Proposition 2.4, the functor $-(H, \varphi)$ takes monomial $\mathcal{O} G$-modules to monomial $\mathcal{O} N_{G}(H, \varphi)$-modules, and it commutes with direct sums. Thus $\bar{V}(H, \varphi) \in$ $k N_{G}(H, \varphi)$ lin and $\overline{\bar{V}}(H, \varphi) \in{ }_{F N_{G}(H, \varphi)}$ lin; in particular, we have $\operatorname{dim}_{F} \overline{\bar{V}}(H, \varphi)=$ $\operatorname{rk}_{k} \bar{V}(H, \varphi)$. In order to prove that $\operatorname{rk}_{k} \bar{V}(H, \varphi)=m_{(H, \varphi)}(V)$ we may certainly assume that $G=H$ and that $U=V \oplus W$ with $U, W \in \mathcal{O}_{G}$ lin and $U$ monomial. Since $\mathcal{O}_{\varphi}(H, \varphi) \cong \mathcal{O}_{\varphi} / p \mathcal{O}_{\varphi}$ has $k$-rank 1 , the additivity of the Brauer construction implies $m_{(H, \varphi)}(V) \leqslant \operatorname{rk}_{k} \bar{V}(H, \varphi)$. Thus, from Proposition 2.6 we obtain

$$
\begin{aligned}
m_{(H, \varphi)}(U) & =m_{(H, \varphi)}(V)+m_{(H, \varphi)}(W) \leqslant \operatorname{rk}_{k} \bar{V}(H, \varphi)+\operatorname{rk}_{k} \bar{W}(H, \varphi) \\
& =\operatorname{rk}_{k} \bar{U}(H, \varphi)=m_{(H, \varphi)}(U) .
\end{aligned}
$$

This shows that $m_{(H, \varphi)}(V)+m_{(H, \varphi)}(W)=\operatorname{rk}_{k} \bar{V}(H, \varphi)+\operatorname{rk}_{k} \bar{W}(H, \varphi)$, and then also $m_{(H, \varphi)}(V)=\operatorname{rk}_{k} \bar{V}(H, \varphi)$.

3.6. Remark. By Bo98, Corollary 2.7], the multiplicities $m_{(H, \varphi)}(V),(H, \varphi) \in$ $\mathcal{M}_{G}(\mathcal{O})$, determine $V \in \mathcal{O}_{G}$ lin up to isomorphism. Therefore, the above theorem implies that also the Brauer constructions $\bar{V}(H, \varphi)$ (resp. $\overline{\bar{V}}(H, \varphi)),(H, \varphi) \in$ $\mathcal{M}_{G}(\mathcal{O})$, and even their ranks (resp. dimensions) determine $V$. The proof of Corollary 2.7 in Bo98] makes use of Proposition 2.6 in the same article. We noticed a gap in the proof of this proposition which will be filled in Section 5 .

3.7. Proposition. Let $V \in{ }_{\mathcal{O} G}$ lin be indecomposable with defect pair $(P, \psi)$. Moreover, let $(H, \varphi) \in \mathcal{M}_{G}(\mathcal{O})$ and let $Q$ be a Sylow p-subgroup of $H$. Then, $\bar{V}(H, \varphi) \neq$ 0 implies that $\left(Q,\left.\varphi\right|_{Q}\right) \leqslant{ }^{g}(P, \psi)$ for some $g \in G$. If $H$ is a $p$-subgroup of $G$, also the converse is true.

Proof. By Theorem 3.5, $\bar{V}(H, \varphi) \neq 0$ is equivalent to $\mathcal{O}_{\varphi} \mid \operatorname{Res}_{H}^{G}(V)$. Since $V \mid$ $\operatorname{Ind}_{P}^{G}\left(\mathcal{O}_{\psi}\right)$, Mackey's decomposition formula shows that $\mathcal{O}_{\varphi} \mid \operatorname{Res}_{H}^{G}(V)$ implies

$$
\mathcal{O}_{\varphi} \mid \operatorname{Ind}_{H \cap g P}^{H}\left(\mathcal{O}_{\left.\left({ }^{g} \psi\right)\right|_{H \cap g_{P}}}\right)
$$

for some $g \in G$. By Lemma 2.5 this implies that $H \cap{ }^{g} P$ is a Sylow $p$-subgroup of $H$ and that $\left.\varphi\right|_{H \cap{ }^{g} P}=\left.{ }^{g} \psi\right|_{H \cap g} P$. Thus $\left(H \cap{ }^{g} P,\left.\varphi\right|_{H \cap{ }^{g} P}\right) \leqslant{ }^{g}(P, \psi)$, and there exists $h \in H$ such that ${ }^{h}\left(H \cap{ }^{g} P\right)=Q$. Then ${ }^{h}\left(H \cap{ }^{g} P,\left.\varphi\right|_{H \cap g} P\right)=\left(Q,\left.{ }^{h} \varphi\right|_{Q}\right)=\left(Q,\left.\varphi\right|_{Q}\right)$. Finally, if $H=Q$ and $\left(Q,\left.\varphi\right|_{Q}\right) \leqslant{ }^{g}(P, \psi)$, then

$$
\mathcal{O}_{\varphi}\left|\operatorname{Res}_{Q}^{g} P\left(\mathcal{O}_{g_{\psi}}\right)\right| \operatorname{Res}_{Q}^{g} P\left(\operatorname{Res}_{g_{P}}^{G}(V)\right),
$$

and $V(H, \varphi) \neq 0$ by Theorem 3.5

3.8. We recall the Green correspondence as explained, for example, in [Th Proposition (20.8)]. Thus let $(P, \psi) \in \mathcal{M}_{G}(\mathcal{O})_{p}$. If $V \in \mathcal{O}_{G}$ lin is indecomposable with defect pair $(P, \psi)$, then $\operatorname{Res}_{N_{G}(P, \psi)}^{G}(V)$ has a unique (up to isomorphism) indecomposable direct summand $W \in \mathcal{O N}_{G}(P, \psi)$ lin with defect pair $(P, \psi), W$ occurs with multiplicity one, and all other indecomposable direct summands $W^{\prime}$ of $\operatorname{Res}_{N_{G}(P, \psi)}^{G}(V)$ have 
defect pairs $(Q, \mu)$ such that $(Q, \mu) \leqslant{ }^{g}(P, \psi)$ for some $g \in G$. It may happen that $Q=P$. Conversely, if $W \in \mathcal{O}_{N_{G}(P, \psi)}$ lin is indecomposable with defect pair $(P, \psi)$, then $\operatorname{Ind}_{N_{G}(P, \psi)}^{G}(W)$ has a unique (up to isomorphism) indecomposable direct summand $V$ with defect pair $(P, \psi), V$ occurs with multiplicity one, and all other indecomposable direct summands $V^{\prime}$ of $\operatorname{Ind}_{N_{G}(P, \psi)}^{G}(W)$ have defect pairs strictly smaller than $(P, \psi)$. In this way one obtains mutually inverse bijections between $\operatorname{IInd}^{(P, \psi)}(\mathcal{O} G)$, the set of isomorphism classes of indecomposable linear source $\mathcal{O} G$ modules with defect pair $(P, \psi)$, and the corresponding set $\operatorname{Ind}^{(P, \psi)}\left(\mathcal{O} N_{G}(P, \psi)\right)$. These bijections are called the Green correspondence with respect to $(P, \psi)$.

3.9. Theorem. Let $V \in \mathcal{O}_{G}$ lin be indecomposable with defect pair $(P, \psi)$, and let $W \in \mathcal{O N}_{G}(P, \psi)$ lin be the Green correspondent of $V$ with respect to $(P, \psi)$. Then

$$
\bar{V}(P, \psi) \cong W / p W \quad \text { and } \quad \overline{\bar{V}}(P, \psi) \cong W / \pi W .
$$

Proof. We write $\operatorname{Res}_{N_{G}(P, \psi)}^{G}(V)=W \oplus W^{\prime}$ with $W^{\prime} \in \mathcal{O}_{N_{G}(P, \psi)}$ lin, and fix an indecomposable direct summand $U$ of $W^{\prime}$. Let $\left(P^{\prime}, \psi^{\prime}\right)$ be a defect pair of $U$. Then $\left(P^{\prime}, \psi^{\prime}\right) \leqslant{ }^{g}(P, \psi)$ for some $g \in G$, and $\left(P^{\prime}, \psi^{\prime}\right) \neq(P, \psi)$ by the remarks above. Thus, by Proposition 3.7 we have $\bar{U}(P, \psi)=0$. Hence $\bar{V}(P, \psi)=\bar{W}(P, \psi)$. On the other hand, Proposition 3.7 implies that $\bar{W}(P, \psi) \neq 0$. Thus $\mathcal{O}_{\psi} \mid \operatorname{Res}_{P}^{N_{G}(P, \psi)}(W)$ by Theorem 3.5, and $W=W^{(P, \psi)}$ by Theorem 3.3. Hence $\bar{W}(P, \psi)=W / p W$, and the result follows.

3.10. Remark. Let $(P, \psi) \in \mathcal{M}_{G}(\mathcal{O})_{p}$. Then by Theorem 3.3 the indecomposable direct summands of $\operatorname{Ind}_{P}^{N_{G}(P, \psi)}\left(\mathcal{O}_{\psi}\right)$ all have defect pair $(P, \psi)$. Thus the set $\operatorname{IInd}^{(P, \psi)}\left(\mathcal{O} N_{G}(P, \psi)\right)$ is in bijection with the set of isomorphism classes of indecomposable projective modules over $E:=\operatorname{End}_{\mathcal{O} N_{G}(P, \psi)}\left(\operatorname{Ind}_{P}^{N_{G}(P, \psi)}\left(\mathcal{O}_{\psi}\right)\right)$. But $E$ is a twisted group algebra of $N_{G}(P, \psi) / P$ over $\mathcal{O}$, and every element in $E$ induces an $F N_{G}(P, \psi)$-endomorphism of

$$
\operatorname{Ind}_{P}^{N_{G}(P, \psi)}\left(\mathcal{O}_{\psi}\right) / \pi \cdot \operatorname{Ind}_{P}^{N_{G}(P, \psi)}\left(\mathcal{O}_{\psi}\right) \cong \operatorname{Ind}_{P}^{N_{G}(P, \psi)}\left(\mathcal{O}_{\psi} / \pi \mathcal{O}_{\psi}\right) \cong \operatorname{Ind}_{P}^{N_{G}(P, \psi)}(F) .
$$

Moreover,

$$
\operatorname{End}_{F N_{G}(P, \psi)}\left(\operatorname{Ind}_{P}^{N_{G}(P, \psi)}(F)\right) \cong F\left[N_{G}(P, \psi) / P\right]
$$

so we obtain $E / \pi E \cong F\left[N_{G}(P, \psi) / P\right]$. Thus reduction modulo $\pi$ yields a bijection between $\operatorname{IInd}^{(P, \psi)}\left(\mathcal{O} N_{G}(P, \psi)\right)$ and $\operatorname{IInd}^{\mathrm{pr}}\left(F\left[N_{G}(P, \psi) / P\right]\right)$, the set of isomorphism classes of indecomposable projective $F\left[N_{G}(P, \psi) / P\right]$-modules. Combining this bijection with Theorem 3.9 we see that the functor $V \mapsto \overline{\bar{V}}(P, \psi)$ induces a bijection

$$
\operatorname{IInd}^{(P, \psi)}(\mathcal{O} G) \rightarrow \operatorname{IInd}^{\mathrm{pr}}\left(F\left[N_{G}(P, \psi) / P\right]\right) .
$$

\section{Reduction of Linear Source Modules}

4.1. We assume the notation established in 3.1. Let $R=\mathcal{O} / \pi^{n} \mathcal{O}$ with $n \in \mathbb{N} \cup$ $\{\infty\}$. We denote by $T_{R}(G) \subseteq L_{R}(G)$ the subrings of the Green ring of $G$ over $R$, generated by the linear source and trivial source $R G$-modules. More precisely, we may consider $L_{R}(G)$ (resp. $T_{R}(G)$ ) as the free abelian group on the isomorphism classes of indecomposable linear source (resp. trivial source) $R G$-modules. Note that $T_{R}$ and $L_{R}$ are Green functors on $G$ (see for example [Bo96] for a definition). 
Lemma 3.2 implies that, for $R^{\prime}=\mathcal{O} / \pi^{m} \mathcal{O}$ with $m \leqslant n$, reduction modulo $\pi^{m}, \pi^{n}$, and $\pi$ induces functors

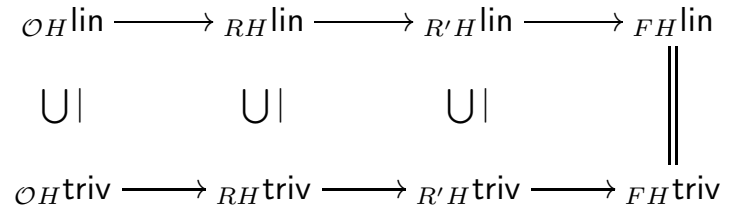

for all $H \leqslant G$ which commute with direct sums, tensor products, restriction, induction and conjugation. Thus, we obtain morphisms of Green functors

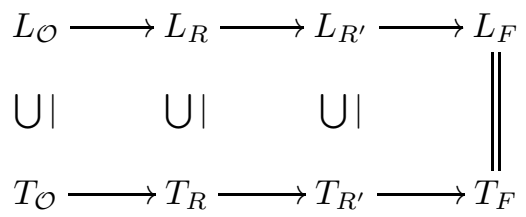

on $G$. Note that for $n=1$ we have $T_{F}=L_{F}$, and recall that the reduction map $T_{\mathcal{O}} \rightarrow T_{F}$ is an isomorphism (see for example $\mathrm{Br}$, (3.5)]) which preserves indecomposablility and vertices. One can also see easily that each map in the bottom row of Diagram (4.1.a) is an isomorphism preserving indecomposability and vertices. In fact, it suffices to show that this is true for $T_{\mathcal{O}} \rightarrow T_{R}$ and any $R=\mathcal{O} / \pi^{n} \mathcal{O}$ with $n \in \mathbb{N}$. By Remark 1.7, for each $p$-subgroup $P$ of $G$, the natural ring homomorphism $\operatorname{End}_{\mathcal{O} G}\left(\operatorname{Ind}_{P}^{G}(\mathcal{O})\right) \rightarrow \operatorname{End}_{R G}\left(\operatorname{Ind}_{P}^{G}(R)\right)$ is surjective with kernel consisting of those endomorphisms $f$ satisfying $f\left(\operatorname{Ind}_{P}^{G}(\mathcal{O})\right) \subseteq \pi^{n} \operatorname{Ind}_{P}^{G}(\mathcal{O})$. Therefore, the kernel is contained in the radical of $\operatorname{End}_{\mathcal{O} G}\left(\operatorname{Ind}_{P}^{G}(\mathcal{O})\right)$ and the lifting theorem of idempotents applies to show that reduction modulo $\pi^{n}$ preserves indecomposability of relatively $P$-projective trivial source modules and induces a bijection between the set of isomorphism classes of indecomposable direct summands of $\operatorname{Ind}_{P}^{G}(\mathcal{O})$ and of $\operatorname{Ind}_{P}^{G}(R)$. Since vertices can only become smaller under reduction, the preservation of vertices under reduction modulo $\pi^{n} \mathcal{O}$ follows from its preservation under reduction modulo $\pi \mathcal{O}$.

4.2. We will study the reduction morphism $L_{\mathcal{O}} \rightarrow L_{R}$ for $R=\mathcal{O} / \pi^{n} \mathcal{O}$ with $n \in \mathbb{N}$ large enough. Note that each element in $R^{\times}$which is congruent to 1 modulo $J(R)$ has a finite $p$-power order. Hence, in general, the reduction map cannot be surjective. However, injectivity of the reduction map is guaranteed for large $n$ by Maranda's theorem (cf. [CR81, § 30]). We proceed to investigate the question of for which $n \in \mathbb{N}$ the reduction map $L_{\mathcal{O}} \rightarrow L_{R}$ is injective in more detail. We start by investigating the reduction of rank one modules.

Recall that the ramification index $e_{\mathbb{Q}_{p}(\zeta) / \mathbb{Q}_{p}}$ of $\mathbb{Q}_{p}(\zeta)$ over $\mathbb{Q}_{p}$, for a primitive $d$-th root of unity $\zeta$, with $d=p^{r} m$ where $p \nmid m$, is given by $p^{r-1}(p-1)$ if $r \geqslant 1$, and by 1 if $r=0$, and that $v_{\mathbb{Q}_{p}(\zeta)}\left(1-\zeta^{m}\right)=1$. Also recall that reduction modulo $\pi \mathcal{O}$ induces an isomorphism between the $p^{\prime}$-part of the group $\mu_{K}$ of roots of unity of $K$ and the corresponding group $\mu_{F}$. So if $\mu_{K}$ is a $p^{\prime}$-group, then reduction modulo $\pi^{n} \mathcal{O}$ is injective for arbitrary $n \in \mathbb{N}$. The remaining case is treated in the following lemma.

4.3. Lemma. Assume that $K$ contains a primitive $p$-th root of unity and let $n \in \mathbb{N}$. Then the reduction map $\mu_{K} \rightarrow\left(\mathcal{O} / \pi^{n} \mathcal{O}\right)^{\times}$is injective if and only if $n>e /(p-1)$, where e denotes the ramification index of $K / \mathbb{Q}_{p}$. 
Proof. Let $1 \neq \zeta \in \mu_{K}$ and write $\zeta=\zeta_{p^{r}} \cdot \zeta_{m}$ with roots of unity $\zeta_{p^{r}}$ and $\zeta_{m}$ of respective orders $p^{r}$ and $m$, where $p \nmid m$. Since $F^{\times}$has no elements of $p$-power order except 1, we have $\zeta_{p^{r}} \equiv 1 \bmod \pi \mathcal{O}$. Thus, if $\zeta_{m} \neq 1$, then $v_{K}(1-\zeta)=0$. If $\zeta_{m}=1$, then with $K^{\prime}:=\mathbb{Q}_{p}(\zeta)$ we have $v_{K}(1-\zeta)=e_{K / K^{\prime}} v_{K^{\prime}}(1-\zeta)=e_{K / K^{\prime}}=$ $e / p^{r-1}(p-1)$. Hence, $\mu_{K} \rightarrow\left(\mathcal{O} / \pi^{n} \mathcal{O}\right)^{\times}$is injective if and only if $v_{K}(1-\zeta)<n$ for each $\zeta \in \mu_{K} \backslash\{1\}$, which in turn is equivalent to $n>e / p^{r-1}(p-1)$ for all $r \in \mathbb{N}$ such that $K$ has a primitive $p^{r}$-th root of unity. Since $e / p^{r-1}(p-1)$ assumes its minimum for $r=1$, the proof is complete.

4.4. Lemma. Let $n \in \mathbb{N}$ be such that $n>e /(p-1)$, and let $R:=\mathcal{O} / \pi^{n} \mathcal{O}$. Let - (resp. ${ }^{\circ}$ ) denote the functor of reducing modulo $\pi^{n} \mathcal{O}$ (resp. $\left.\pi \mathcal{O}\right)$. Then for each $(H, \varphi) \in \mathcal{M}_{G}(\mathcal{O})$, the rings $\operatorname{End}_{\mathcal{O} G}\left(\operatorname{Ind}_{H}^{G}\left(\mathcal{O}_{\varphi}\right)\right)$ and $\operatorname{End}_{R G}\left(\operatorname{Ind}_{H}^{G}\left(R_{\bar{\varphi}}\right)\right)$ have ideals contained in their respective radicals such that the corresponding factor rings are isomorphic to $F \otimes_{\mathcal{O}} \operatorname{End}_{\mathcal{O} G}\left(\operatorname{Ind}_{H}^{G}\left(\mathcal{O}_{\varphi}\right)\right)$.

Proof. By Remark 1.7 we have a commutative diagram

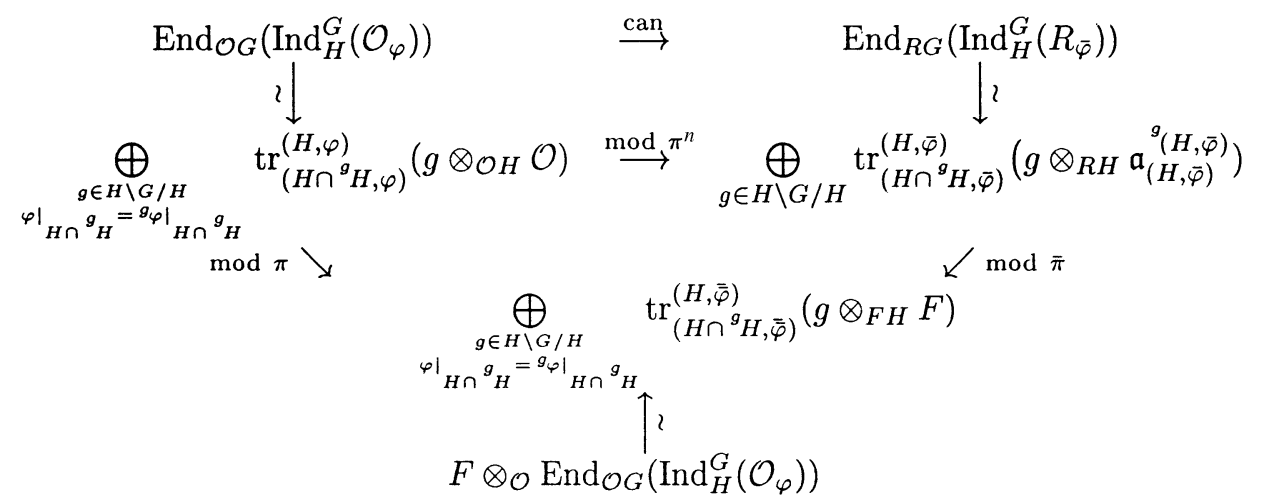

where the upper horizontal map is the obvious natural ring homomorphism. Note that the right diagonal map sends to zero those summands which are parametrized by $g \in H \backslash G / H$ with $\left.\varphi\right|_{H \cap{ }^{g} H} \neq\left.{ }^{g} \varphi\right|_{H \cap{ }^{g} H}$, since, by Lemma4.3, we have $\mathfrak{a}_{(H, \bar{\varphi})}^{{ }^{g}(H, \bar{\varphi})}=R$ if and only if $\left.\varphi\right|_{H \cap{ }^{g} H}=\left.{ }^{g} \varphi\right|_{H \cap{ }^{g} H}$.

Obviously both diagonal maps are surjective, the kernels consisting of those endomorphisms whose images are contained in $\pi \operatorname{Ind}_{H}^{G}\left(\mathcal{O}_{\varphi}\right)$, resp. $\bar{\pi} \operatorname{Ind}_{H}^{G}\left(R_{\bar{\varphi}}\right)$. Therefore, the kernels are ideals contained in the respective radicals. Obviously the left diagonal map is a ring homomorphism, since it is induced by the canonical map $\operatorname{End}_{\mathcal{O} G}\left(\operatorname{Ind}_{H}^{G}\left(\mathcal{O}_{\varphi}\right)\right) \rightarrow F \otimes_{\mathcal{O}} \operatorname{End}_{\mathcal{O} G}\left(\operatorname{Ind}_{H}^{G}\left(\mathcal{O}_{\varphi}\right)\right)$. Since the triangle commutes, since $\operatorname{End}_{R G}\left(\operatorname{Ind}_{H}^{G}\left(R_{\bar{\varphi}}\right)\right)$ is the sum of the image of the horizontal map and the kernel of the right diagonal map, and since the left diagonal map is a ring homomorphism, also the right diagonal map is a ring homomorphism, and the proof is complete.

4.5. Theorem. Let $n \in \mathbb{N}$ be such that $n>e /(p-1)$, let $R:=\mathcal{O} / \pi^{n} \mathcal{O}$, let . denote reduction modulo $\pi^{n} \mathcal{O}$, and let $V \in{ }_{\mathcal{O}} G_{\text {lin }}$ be indecomposable with defect pair $(P, \psi) \in \mathcal{M}_{G}(\mathcal{O})_{p}$ and with Green correspondent $W \in \mathcal{O N}_{G}(P, \psi)$ lin with respect to $(P, \psi)$.

(a) The reduction $\bar{V} \in{ }_{R G}$ lin of $V$ is indecomposable.

(b) The pair $(P, \bar{\psi})$ is a defect pair of $\bar{V}$, and $N_{G}(P, \psi)=N_{G}(P, \bar{\psi})$. 
(c) The module $\bar{W} \in R_{R N_{G}(P, \psi)}$ lin is the Green correspondent of $\bar{V}$ with respect to $(P, \bar{\psi})$.

(d) Reduction modulo $\pi^{n} \mathcal{O}$ induces a bijection $\operatorname{IInd}^{(P, \psi)}(\mathcal{O} G) \rightarrow \operatorname{IInd}^{(P, \bar{\psi})}(R G)$.

(e) Two modules $X, X^{\prime} \in \mathcal{O}_{G}$ lin are isomorphic if and only if their reductions $\bar{X}, \bar{X}^{\prime} \in R_{\text {G lin }}$ are isomorphic.

Proof. (a) This follows immediately from Lemma 4.4 .

(b), (c) By Lemma 4.3 we have $N_{G}(P, \psi)=N_{G}(P, \bar{\psi})$, and by part (a) the module $\bar{W}$ is indecomposable. Moreover, it is easy to see that $\bar{W}$ has defect pair $(P, \bar{\psi})$, since $\bar{W} \mid \operatorname{Ind}_{P}^{N_{G}(P, \psi)}\left(\mathcal{O}_{\bar{\psi}}\right)$. Hence, its Green correspondent is the unique indecomposable direct summand of $\operatorname{Ind}_{N_{G}(P, \psi)}^{G}(\bar{W})$ with defect pair $(P, \bar{\psi})$. Since

$$
\operatorname{Ind}_{N_{G}(P, \psi)}^{G}(\bar{W}) \cong \overline{\operatorname{Ind}_{N_{G}(P, \psi)}^{G}(W)} \cong \overline{V \oplus V^{\prime}} \cong \bar{V} \oplus \overline{V^{\prime}}
$$

with $V^{\prime} \in \mathcal{O}_{G}$ lin having only indecomposable direct summands with vertex of order smaller than $|P|$, the module $\bar{V}$ has to be the Green correspondent of $\bar{W}$ with respect to $(P, \bar{\psi})$ and has the same defect pair $(P, \bar{\psi})$ as $\bar{W}$.

(d) This follows from part (b) and Lemma 4.4

(e) This follows from part (c) and part (d).

4.6. Corollary. Let $V \in \mathcal{O} G$ lin. The reduction map $V \mapsto V / p V$ for $p \geqslant 3$ and $V \mapsto V / 4 V$ for $p=2$ induces an injective ring homomorphism $L_{\mathcal{O}}(G) \rightarrow L_{\mathcal{O} / p \mathcal{O}}(G)$ for odd $p$, resp. $L_{\mathcal{O}}(G) \rightarrow L_{\mathcal{O} / 4 \mathcal{O}}(G)$ for $p=2$, which preserves indecomposability and vertices.

4.7. Remark. If $V \in \mathcal{O}_{G}$ lin is indecomposable, then in general $V / \pi V$ need not be indecomposable: Let $G=S_{3}$, the symmetric group of degree 3 , let $p=3$, and let $P \triangleleft G$ denote the Sylow $p$-subgroup. Moreover, let $\psi \in \hat{P}(\mathcal{O})$ be non-trivial. Then $V:=\operatorname{Ind}_{P}^{G}\left(\mathcal{O}_{\psi}\right)$ is indecomposable, since its character is irreducible. But $V / \pi V \cong \operatorname{Ind}_{P}^{G}(F)$ splits into two one-dimensional $F G$-modules.

4.8. Remark. The collection of the functors ${ }^{\circ}(P, \psi)$ for $(P, \psi) \in \mathcal{M}_{G}(\mathcal{O})_{p}$ induces a map

$$
\begin{aligned}
\alpha: L_{\mathcal{O}}(G) \rightarrow & \left(\prod_{(P, \psi) \in \mathcal{M}_{G}(\mathcal{O})_{p}} T_{F}\left(N_{G}(P, \psi) / P\right)\right)^{G} \\
& \cong \prod_{(P, \psi) \in \mathcal{M}_{G}(\mathcal{O})_{p} / G} T_{F}\left(N_{G}(P, \psi) / P\right),
\end{aligned}
$$

where $G$ acts on the product by conjugation. Note that after ordering $\mathcal{M}_{G}(\mathcal{O})_{p} / G$ according to the orders of the involved $p$-subgroups $P$, and ordering the indecomposable linear source modules in a similar way according to their defect pairs, Proposition 3.7 implies that the matrix associated to the map $\alpha$ is a block triangular matrix. The block with coordinates $(P, \psi)$ and $(Q, \varphi)$ from a set of representatives for the $G$ conjugacy classes of $\mathcal{M}_{G}(\mathcal{O})_{p}$ is indexed by $\operatorname{IInd}^{(P, \psi)}(\mathcal{O} G) \times \operatorname{IInd}\left(F N_{G}(Q, \varphi) / Q\right.$ triv $)$, where the latter factor denotes the set of isomorphism classes of indecomposable trivial source $F N_{G}(Q, \varphi) / Q$-modules. Associating to each trivial source module its Brauer character induces a map

$$
\prod_{(P, \psi) \in \mathcal{M}_{G}(\mathcal{O})_{p} / G} T_{F}\left(N_{G}(P, \psi) / P\right) \rightarrow \prod_{(P, \psi) \in \mathcal{M}_{G}(\mathcal{O})_{p} / G} R_{F}\left(N_{G}(P, \psi) / P\right)
$$


which composes with $\alpha$ to a map

$$
\beta: L_{\mathcal{O}}(G) \rightarrow \prod_{(P, \psi) \in \mathcal{M}_{G}(\mathcal{O})_{p} / G} R_{F}\left(N_{G}(P, \psi) / P\right)
$$

mapping the class of a linear source $\mathcal{O} G$-module $V$ to the family of Brauer characters of $\overline{\bar{V}}(P, \psi),(P, \psi) \in \mathcal{M}_{G}(\mathcal{O})_{p} / G$. Here, by $R_{F}(G)$ we denote the Grothendieck group of the category of finitely generated $F G$-modules. The matrix representing the map $\beta$ is again block triangular with the Cartan matrix of $N_{G}(P, \psi) / P$ as diagonal block at position $(P, \psi)$. Thus $\beta$ is injective and invertible after tensoring with $\mathbb{Q}$.

\section{A CORRECTION}

We conclude this article by filling a gap in the proof of Proposition 2.6 in Bo98. The proof there only reduced the statement of Proposition 2.6 to the proof of the following proposition. The following notation is needed. Let $L_{\mathcal{O}}^{\prime}(G) \subseteq L_{\mathcal{O}}(G)$ denote the span of the isomorphism classes of those indecomposable $V \in \mathcal{O}_{G}$ lin which have the Sylow $p$-subgroups of $G$ as vertices, and let $q_{G}: L_{\mathcal{O}}(G) \rightarrow L_{\mathcal{O}}^{\prime}(G)$ denote the obvious projection map. A finite group $G$ is called $p$-hypoelementary if it has a normal $p$-subgroup with cyclic factor group.

5.1. Proposition. Let $G$ be a p-hypoelementary group and assume that $\mathcal{O}$ contains a root of unity of order $|G|$. Let $x \in L_{\mathcal{O}}(G)$ be an element such that $q_{H}\left(\operatorname{res}_{H}^{G}(x)\right)=0$ for all $H \leqslant G$. Then $x=0$.

Proof. Let $V, W \in \mathcal{O}_{G}$ lin be given such that, for all subgroups $H \leqslant G$, each indecomposable $\mathcal{O} H$-module with the Sylow $p$-subgroup of $H$ as vertex occurs with the same multiplicity as direct summand in $\operatorname{Res}_{H}^{G}(V)$ and in $\operatorname{Res}_{H}^{G}(W)$. It suffices to show that in this situation we have $V \cong W$. We will prove this by induction on the order of $G$ and then by induction on $\operatorname{rk}_{\mathcal{O}} V+\operatorname{rk}_{\mathcal{O}} W$.

We choose among all indecomposable direct summands of $V$ and $W$ one, say $X$, with maximal vertex $Q$, and assume by symmetry that $X \mid V$. Let $(Q, \psi)$ be a defect pair of $X$, let $N:=N_{G}(Q)$, and let $M:=N_{G}(Q, \psi)$.

Assume that $N<G$. Then by induction $\operatorname{Res}_{N}^{G}(V) \cong \operatorname{Res}_{N}^{G}(W)$. Let $X^{\prime} \in \mathcal{O}_{N}$ lin denote a Green correspondent of $X$. So also $X^{\prime} \mid \operatorname{Res}_{N}^{G}(W)$. Let $Y \mid W$ be indecomposable such that $X^{\prime} \mid \operatorname{Res}_{N}^{G}(Y)$. Then the maximality of $Q$ and the Green correspondence imply that $Y \cong X$. Hence, $V \cong X \oplus V^{\prime}$ and $W \cong Y \oplus W^{\prime}$ for some $V^{\prime}, W^{\prime} \in \mathcal{O}_{G}$ lin. Then the hypothesis at the beginning of the proof is also satisfied for $V^{\prime}$ and $W^{\prime}$, and by induction on the ranks we have $V^{\prime} \cong W^{\prime}$, hence $V \cong W$.

Therefore we assume from now on that $N=G$. Assume further that $M<$ $G$. Then $\operatorname{Res}_{M}^{G}(V) \cong \operatorname{Res}_{M}^{G}(W)$ by induction. By Theorem 3.3 we know that $X^{(Q, \psi)} \mid \operatorname{Res}_{M}^{G}(X)$ and $X \cong \operatorname{Ind}_{M}^{G}\left(X^{(Q, \psi)}\right)$. Let $Y \mid W$ be an indecomposable direct summand such that $X^{(Q, \psi)} \mid \operatorname{Res}_{M}^{G}(Y)$. Then, again by Theorem 3.3 we have $X^{(Q, \psi)} \cong Y^{(Q, \psi)}$, and then $X \cong Y$. Therefore we have $V \cong W$ by induction on the ranks as before.

From now on we assume that $M=G$. Let $I:=\operatorname{ker} \psi$, let $P \unlhd G$ be the Sylow $p$-subgroup of $G$, and let $C \leqslant G$ be a complement of $P$ in $G$, hence a cyclic $p^{\prime}$ subgroup. Since $(Q, \psi)$ is stable under $G$, it follows that the subgroups $Q$ and $I$ are normal in $G$, and $G$ centralizes the factor group $Q / I$. We set $H=Q C$. Then $H / I$ decomposes as the direct product of $C I / I \cong C$ and $Q / I$. Let $\tilde{\psi} \in \hat{H}(\mathcal{O})$ be 
such that $\left.\tilde{\psi}\right|_{Q}=\psi$ and $\left.\tilde{\psi}\right|_{C}=1$. Moreover, let $\varphi_{1}, \ldots, \varphi_{r} \in \hat{H}(\mathcal{O})$ denote those homomorphisms which are trivial on $Q$. Then $r=|C|$ and $\varphi_{i} \tilde{\psi}, i=1, \ldots, r$, are the different extensions of $\psi$ to $H$. Therefore we have

$$
\operatorname{Ind}_{Q}^{G}\left(\mathcal{O}_{\psi}\right) \cong \operatorname{Ind}_{H}^{G}\left(\operatorname{Ind}_{Q}^{H}\left(\mathcal{O}_{\psi}\right)\right) \cong \bigoplus_{i=1}^{r} \operatorname{Ind}_{H}^{G}\left(\mathcal{O}_{\varphi_{i} \tilde{\psi}}\right) .
$$

The summands $X_{i}:=\operatorname{Ind}_{H}^{G}\left(\mathcal{O}_{\varphi_{i} \tilde{\psi}}\right), i=1, \ldots, r$, are indecomposable, since $\operatorname{Res}_{P}^{G}\left(X_{i}\right) \cong \operatorname{Ind}_{Q}^{P}\left(\mathcal{O}_{\psi}\right)$ is indecomposable. Let $m_{i}$, resp. $n_{i}, i=1, \ldots, r$, denote the multiplicities of $X_{i}$ as direct summands in $V$, resp. $W$. Note that any indecomposable module $Z \in \mathcal{O}_{G}$ lin such that $\mathcal{O}_{\psi} \mid \operatorname{Res}_{Q}^{G}(Z)$ is isomorphic to $X_{i}$ for some $i=1, \ldots, r$, by the maximality of $Q$ and by Theorem 3.3 (a). Thus $X \cong X_{j}$ for some $j \in\{1, \ldots, r\}$, and $m_{j}>0$ for this $j$. By our hypothesis we therefore know that

$$
\sum_{i=1}^{r} m_{i} \operatorname{res}_{H}^{G}\left(\left[X_{i}\right]\right)=\sum_{i=1}^{r} n_{i} \operatorname{res}_{H}^{G}\left(\left[X_{i}\right]\right) \in L_{\mathcal{O}}^{\prime}(H) .
$$

Here, $\left[X_{i}\right]$ denotes the element in $L_{\mathcal{O}}(G)$ associated to $X_{i}$. Now it suffices to show that the elements $\operatorname{res}_{H}^{G}\left(\left[X_{i}\right]\right), i=1, \ldots, r$, are linearly independent in $L_{\mathcal{O}}^{\prime}(H)$. Let $X_{i}^{\prime}:=\operatorname{Ind}_{H}^{G}\left(\mathcal{O}_{\varphi_{i}}\right)$ for $i=1, \ldots, r$. Then $\operatorname{Res}_{H}^{G}\left(X_{i}^{\prime}\right) \cong \mathcal{O}_{\tilde{\psi}^{-1}} \otimes \operatorname{Res}_{H}^{G}\left(X_{i}\right)$, and it suffices to show that the elements $\operatorname{res}_{H}^{G}\left(\left[X_{i}^{\prime}\right]\right), i=1, \ldots, r$, are linearly independent in $L_{\mathcal{O}}^{\prime}(H)$. We have $\operatorname{Ind}_{Q}^{G}(\mathcal{O}) \cong X_{1}^{\prime} \oplus \cdots \oplus X_{r}^{\prime}$ and $X_{1}^{\prime}, \ldots, X_{r}^{\prime}$ are indecomposable by the same argument as for $X_{1}, \ldots, X_{r}$. Since $Q$ acts trivially on $X_{1}^{\prime}, \ldots, X_{r}^{\prime}$, we may assume that $Q=1$. Then $X_{1}^{\prime}, \ldots, X_{r}^{\prime}$ are all the projective indecomposable modules of $G$. They are pairwise distinct, since $F G$ has precisely $r$ simple modules. It is well-known that the characters $\chi_{i}^{\prime}$ of $X_{i}^{\prime}, i=1, \ldots, r$, are linearly independent and vanish on $p$-singular classes. Therefore, the restrictions of $\chi_{1}^{\prime}, \ldots, \chi_{r}^{\prime}$ to the $p^{\prime}$-Hall subgroup $H$ of $G$ are still linearly independent, since all $p^{\prime}$-Hall subgroups of $G$ are conjugate. This completes the proof.

\section{REFERENCES}

[Be] D. Benson: Representations and cohomology, vol. I. Cambridge University Press, Cambridge 1991. MR 92m:20005

[Bo96] R. Boltje: A general theory of canonical induction formulae. J. Algebra 206 (1998), 293343. MR 99k:20013

[Bo98] R. Boltje: Linear source modules and trivial source modules. Proc. Sympos. Pure Math. 63 (1998), 7-30. MR 99d:20016

[Br] M. Broué: On Scott modules and p-permutation modules: An approach through the Brauer morphism. Proc. Amer. Math. Soc. 93 (1985), 401-408. MR 86d:20010

[CR81] C.W. Curtis, I. Reiner: Methods of representation theory, Vol. 1. J. Wiley \& Sons, New York 1981. MR 82i:20001

[CR87] C.W. Curtis, I. Reiner: Methods of representation theory, Vol. 2. J. Wiley \& Sons, New York 1987. MR 88f:20002

[Gr] J.A. Green: On the indecomposable representations of a finite group. Math. Zeitschr. 70 (1959), 430-445. MR 24:A1304

[Th] J. Thévenaz: $G$-algebras and modular representation theory. Oxford University Press 1995. MR 96j:20017

Department of Mathematics, University of California, Santa Cruz, California 95064

E-mail address: boltje@math.ucsc.edu

Mathematisches Institut, Universität Jena, 07740 Jena, Germany

E-mail address: kuelshammer@uni-jena.de 